\title{
INDIVIDUALISMO METODOLÓGICO Y LIBERALISMO
}

\author{
MANUEL HERRERA GÓMEZ \\ Universidad de Granada
}

\author{
PALABRAS CLAVE ADICIONALES \\ Epistemología de las Ciencias Sociales, Filosofia \\ y Metodología de las Ciencias Sociales, Teoría \\ Sociológica. \\ ADDITIONAL KEYWORDS \\ Epistemology of Social Sciences, Philosophy \\ and Methodology of Social Sciences, Social \\ Theory.
}

RESUMEN. Para los miembros de la Escuela Austriaca, las ciencias sociales tienen como objetivo elaborar una teoría de la acción humana en condiciones de escasez, y no es difícil identificar en ellos la referencia a la teoría de los valores subjetivos del marginalismo austriaco. Desde el punto de vista de la filosofía de las ciencias sociales, dicha teoría puede ser contemplada como un intento de explicar los fenómenos y las instituciones sociales como consecuencias involuntarias de acciones humanas dotadas de un conocimiento limitado y erróneo. Por otra parte, conviene recordar que tales instituciones evolucionan como consecuencia de la interacción con los individuos con los que se relacionan. La tesis que se desarrolla en este artículo es la siguiente: para los máximos representantes de la Escuela Austriaca, el individualismo metodológico no sólo se configura como un canon explicativo y metodológico, sino que es la premisa de una filosofia de las ciencias sociales y de una filosofia política.

\begin{abstract}
In opinion of the chief representatives of the Austrian School, the objetive of the theoretical social sciences is to elaborate a theory of human action under shortage conditions, while it is not difficult to particularize the reference to the theory of the subjective values of the Austrian marginalism. This theory, under the point of view of the social sciences philosophy, can be seen as an attempt to explain the social phenomenon and institutions as involuntary consequences of human actions endued with a limited and wrong knowledge. On the other hand, we must recall that such institutions have evolved deriving from the interaction with the individuals which they are related. This article's thesis develops as follows: in opinion of the chief representatives of the Austrian School, the methodological individualism is not only configurated as an explicative and methodological standard, but as the premise of a social sciences and political philosophy, trying to reveal their theoretical groundwork.
\end{abstract}

E-mail: manuel.herrera@educ.mec.es; mherrera@goliat.ugr.es

Revista Internacional de Sociología (RIS)

Tercera Época, $n^{\circ} 34$, Enero-Abril, 2003, pp. 7-32. 
RIS

REVISTA INTERNACIONAL DE SOCIOLOCIA

№ 34, Enero-Abril, 2003

MANUEL HERRERA GÓMEZ

\section{INTRODUCCIÓN}

Como muchas ideas fecundas, el concepto de individualismo metodológico posee una vida casi autónoma y, en consecuencia - no se sabe bien si, por simple incomprensión o ignorancia del significado originario, o incluso por mala fe-, ha derivado en significados diferentes del originario. El resultado es que a veces es presentado como un proceso reductivo, otras veces, como un modelo de explicación a través de una mano invisible, y otras como un mero modelo explicativo sin conexión concreta con una precisa filosofia política.

Cronológicamente, la primera interpretación errónea es la que considera al individualismo metodológico como un método reductivo. Recientemente se ha difundido la tendencia a considerarlo en relación con la smithiana mano invisible (explicación de la mano invisible). Sin embargo, es preciso recordar que la mano invisible es para Smith sinónimo de Providencia, y que los fenómenos sociales presentan mecanismos auto-correctores, análogos a las leyes de la naturaleza, que actúan de forma independiente respecto a las voluntades individuales.

En el caso de la concepción de individualismo metodológico de Friedrich A. Von Hayek y de Karl R. Popper no se puede afirmar que siga el mismo proceso, en cuanto que, incluso en este caso, las acciones individuales y colectivas tienen efectos que pueden ser, en buena medida, independientes de las acciones originarias. Los fenómenos y las instituciones sociales que se quieren explicar a través del individualismo metodológico son efectos de una socialmente difusa insuficiencia de expectativas subjetivas que son fruto de una selección cultural. En consecuencia, el orden, aun siendo un producto espontáneo, no es un producto natural, sino cultural. Dicho en otros términos, no se realiza naturalmente, e incluso puede no realizarse. En la teoría del nacimiento de las instituciones humanas formulada por Hayek y por Popper no existe una mano invisible que orienta hacia una particular configuración; incluso si tal configuración es resultado de un proceso que, aun teniendo una componente genética (conectada a la naturaleza del fenómeno) es, en buena medida, imprevisible. Ni Hayek, ni Popper piensan en términos de selección natural, y tampoco piensan (al contrario de cuantos adoptan la expresión mano invisible) en introducir la Providencia en los acontecimientos sociales.

Por ello, más que en lo ineludible del orden social, Hayek y Popper centran la atención en su fragilidad y casualidad, pero también en que si el orden fuese un producto natural, no sería susceptible de una valoración crítica. Al contrario, los fenómenos sociales y las instituciones sociales deben ser, y son, sometidas a una crítica racional, teniendo en cuenta que la razón es, en sí misma, criticable (racionalismo crítico). El evolucionismo, parte integrante de su filosofia de las ciencias sociales, no presupone un proceso finalista e ineludible, sino, y usando un término de Popper, un universo abierto y modificable. Sin embargo, esto no significa que toda volición subjetiva deba realizarse. 
Para argumentar cómo estas interpretaciones no son aceptables, es oportuno aludir a lo que sus primeros teóricos (Hayek y Popper) entienden a partir de la expresión anteriormente señalada, y analizar aquello que Hayek considera que es el objeto de las ciencias sociales, es decir, el estudio de los resultados involuntarios de las acciones que siguen al modo en que los individuos entienden hechos, instituciones y teorías.

Si recorremos rápidamente la historia del concepto, es necesario señalar que hasta la segunda mitad de los años cuarenta no se habla de individualismo metodológico. La expresión no fue acuñada por Carl Menger, sino por Joseph Schumpeter en 1908 en Das Wesen und der Hauptinhalt der theoretischen Nationalökonomie (Schumpeter, 1908: 88-89) y retomada por Max Weber en Wirtschaft und Gessellschaft, obra póstuma publicada en 1922. No aparecen otros usos de la expresión hasta el ensayo de Hayek Scientism and the Study of Society, del 1942-44. En The Poverty of Historicism y en The Open Society and Its Enemies, que aparecen entre 1944 y 1945, será cuando Popper asuma la expresión; sin embargo, no se refiere al uso que habían hecho Schumpeter y Weber, sino a la acepción de Hayek, que retoma el concepto mengeriano. El mismo Menger es citado por Popper.

Si consideramos que la primera versión de The Poverty of Historicism aparece en la misma revista, Economica, en la que había sido publicado el ensayo de Hayek, nos encontramos ante una muy buena indicación del significado que Popper atribuía a la expresión y a la influencia que sobre su metodología de las ciencias sociales teóricas habían tenido Hayek $\mathrm{y}$, de forma indirecta, la problemática Escuela Austriaca. Obviamente no se trata de una influencia en sentido único: trazos de la falsación popperiana en la epistemología hayekiana pueden encontrarse en Economics and Knowldge de 1937.

Respecto a la fortuna de la expresión "individualismo metodológico" en Popper, es cierto que en obras posteriores ya no aparece, pero también es verdad que el concepto está presente en las expresiones "análisis situacional", "lógica de la situación", "principio de racionalidad" y en World 3, expresión en la que se representa, con palabras diversas, el tema fundamental de la teoría austríaca de la comprensión y de la explicación de la acción humana.

Para los exponentes de la Escuela Austriaca, como para Popper, las ciencias sociales teóricas tienen como objetivo elaborar una teoría de la acción humana en condiciones de escasez, y no es dificil identificar en ellos la referencia a la teoría de los valores subjetivos del marginalismo austríaco. Ésta, desde el punto de vista de la filosofia de las ciencias sociales, puede ser contemplada como un intento para explicar los fenómenos y las instituciones sociales como consecuencias involuntarias de acciones humanas dotadas de un conocimiento limitado y erróneo ${ }^{1}$. Por otra parte, conviene recordar que tales instituciones evolucionan

${ }^{1}$ Sobre los diferentes aspectos de la Escuela Austríaca, véase Cubeddu (1993). 
RIS

REVISTA INTERNACIONAL DE SOCIOLOCIA

№ 34, Enero-Abril, 2003

MANUEL HERRERA CÓMEZ

como resultado de la interacción con los individuos con los que se relacionan.

Mi tesis, por tanto, es que en la acepción "austriaca", el individualismo metodológico no sólo se configura como un canon explicativo y metodológico, sino que es la premisa de una filosofia de las ciencias sociales y de una filosofia política, de la que trataré de revelar los fundamentos teóricos. Igualmente importante - tomando como punto de partida a Menger- es precisar que el individualismo metodológico no es un intento de aplicación del método de la economía a las ciencias sociales teóricas. Si el principal objetivo de éstas es explicar "cómo es posible que instancias destinadas a servir al bienestar colectivo, y vitales para su desarrollo, surgen sin una voluntad colectiva encaminada a su creación" (Menger, 1883: 111-112), entre estas "instancias" Menger ubicará no sólo a la moneda, al mercado y a los precios, también al derecho, al lenguaje, al Estado y a la religión. En otros términos, al complejo campo de las ciencias sociales.

En consecuencia, incluso si podemos definir la problemática de la Escuela Austríaca como un intento por ver si la teoría de la acción humana, revelada a partir del descubrimiento del principio de utilidad marginal, puede extenderse a otras áreas de las ciencias sociales, la tesis de la hegemonía de la ciencia económica carece de fundamento. La frase de Menger, en realidad, puede, y debe, entenderse como la afirmación de que todas las instituciones sociales son el resultado involuntario de la acción humana que subyace a las condiciones de escasez, ignorancia y limitación del conocimiento, deduciendo de ello lo imprevisible de los resultados de las acciones (Cubeddu, 1986: 23-45). Nos encontramos ante una teoría de la acción humana válida para todas las ciencias sociales teóricas, y esto comporta también que no existen ciencias superiores y ciencias inferiores, ciencias que se ocupan de fines y ciencias que se ocupan de medios; o sea, no existe una jerarquía de las ciencias, sino que sólo existen clasificaciones convencionales de la acción humana que pueden darse según los intereses cognitivos. La misma acción, en otras palabras, puede ser contemplada e investigada en su sentido económico, ético, político, jurídico, etc., según el significado que le atribuye el agente, o según el punto de vista que interesa a su intérprete.

En fin, trataré de mostrar cómo, internamente a la misma Escuela Austríaca, el principio y fundamento del individualismo metodológico no se ha entendido de forma unívoca.

\section{EL INDIVIDUALISMO METODOLÓGICO: LAS PREMISAS GNOSE OLÓGICAS DE LA ESCUELA AUSTRIACA}

\section{Orígenes del individualismo metodológico}

La primera formulación indirecta del principio del método compositivo, o individualismo metodológico, la podemos encontrar en el "Prefacio" a Grundsätze der 
Volkswirthschaftslehre de 1871, donde Menger, aludiendo al método adoptado, escribe:

"Nos esforzamos en reconducir los más complejos fenómenos de la economía humana a sus elementos más simples aun accesibles a la observación segura, en adaptar a ellos el criterio de valoración correspondiente a su naturaleza, y posteriormente, sirviéndonos de esto, en examinar nuevamente de qué forma los fenómenos económicos más complejos se desarrollan conforme a leyes" (Menger, 1871: VII).

Tal afirmación es importante no sólo porque aparece en el "Prefacio", y volverá a aparecer en los mismos términos en Untersuchungen, sino también porque es representativa de la problemática filosófica ý metodológica mengeriana.

En 1942, la metodología mengeriana "de las ciencias sociales y, en concreto, de la economía política", como especifica el subtítulo de Untersuchungen, es retomada explícitamente por Hayek en Scientism and the Study of Society. En la versión hayekiana, el "método individualista y compositivo" de las ciencias sociales tiene su núcleo en la afirmación de que los hechos sociales no son hechos empíricos, sino elaboraciones conceptuales producidas por los individuos o por entidades colectivas. Por consiguiente es necesario "abstenerse coherentemente de catalogar como hechos a estas entidades abstractas y tomar sistemáticamente las muestras de las concepciones desde las que los individuos son inducidos a la acción: de ahí que el individualismo metodológico esté intimamente conectado con el subjetivismo de las ciencias sociales" (Hayeck, 1952: 39-41).

Dejaré por un momento estas dos primeras formulaciones del principio del método compositivo o individualista, para centrarme en la relación entre individualismo metodológico e individualismo político. La crítica de Menger al "atomismo" y al "pragmatismo" del "liberalismo racionalista unilateral", y la crítica de Hayek al "racionalismo constructivista" del "falso individualismo", son suficientes para aclarar cómo el individualismo metodológico no es concebido como una variante del individualismo político de derivación constructivo-racionalista, sino como una forma diversa de acercarse a los fenómenos sociales y al mundo de la política. Ni se puede decir que ambos tipos de individualismo se ubiquen en la estela de la tradición liberal en cuanto que, para los austriacos, el "racionalismo constructivista" del individualismo "continental", al igual que el cientificismo, es situado como fundamento de la tradición socialista y del moderno constructivismo utilitarista.

Desde la perspectiva de los austriacos podemos definir el tradicionalmente denominado "individualismo político" como una concepción de la sociedad y de la política que tiene como punto de partida la consideración de los individuos como átomos que deliberadamente proyectan las instituciones sociales como instrumentos para conseguir los fines específicos para los que han sido conce- 
RIS

REVISTA INTERNACIONAL DE SOCIOLOCIA

№ 34, Enero-Abril, 2003

MANUEL HERRERA CÓMEZ

bidas. Por contra, el individualismo metodológico tiene como punto de partida una concepción de los individuos como entidades sociales, y presenta a las instituciones como el resultado involuntario de acciones humanas que se proponen resolver problemas con los instrumentos limitados de su conocimiento ${ }^{2}$. Se trata, como intentaré mostrar, de un tipo de individualismo que se opone a la tradición del individualismo racional-constructivista (del que la tradición liberal puede ser considerada una continuación), a la que censura el ser, en realidad, una variedad del socialismo, y de tener poco en común con la auténtica tradición liberal. Ésta, más que entre los dos extremos del "intervencionismo constructivista" guiado por el Estado y por la tradición del "orden espontáneo", deja espacio, en el interior de esta última, entre una tradición conservadora (como, por ejemplo, la de Michael Oakeshott), y una tradición libertaria (por ejemplo, la de Murray N. Rothbard).

Por tanto, el regreso a Menger es necesario, y no sólo debido a la gran variedad de significados que se han atribuido a la expresión.

En Grundsätze y en Untersuchungen, Menger, aunque explica el concepto, no lo adopta, y sólo en las notas manuscritas sobre la recensión de Schmoller a Untersuchungen, corrige - como escribe Hayek en Scientism and the Study of Society - el término "deductivas" con el término "compositivas", aludiendo al propio método (Hayek, 1942: 44-287). Además, en el texto mengeriano es evidente la influencia de la filosofía aristotélica. Hasta el punto de que en las páginas de Untersuchungen en que aborda el método de las "ciencias sociales teóricas", y en concreto cuando define el procedimiento, hay una definición del propio método que hace pensar en una decisiva influencia de lo escrito por Aristóteles en la Politica. Escribe Aristóteles:

"Como en otros campos es necesario analizar la composición hasta llegar a los elementos más simples (es decir, a las partes más pequeñas del todo), y examinando de que elementos resulta, veremos mejor las diferentes capas en que se diferencian el uno del otro y si es posible obtener cualquier noción científica de alguno de ellos. Si se estudiasen las cosas desde su origen y evoluciones, obtendríamos una visión más clara"3.

\footnotetext{
${ }^{2}$ Sobre este tema véanse las críticas de Menger a la "concepción pragmática" del origen de las instituciones sociales y al "liberalismo racionalista unilateral" en Menger (1883:136-137) y Hayek (1948a). Envío de nuevo a esta obra, y a los ensayos "Kinds of Rationalism", en Hayek (1967a), y "The Errors of Constructivism" y "Liberalism" en Hayek (1978a), para una más concreta definición de los dos tipos de individualismo y para una más analítica especificación de los pensadores que se encuentran en esta ubicación.

${ }^{3}$ Aristóteles, Politica, I, 1, 19-24; 2, 24-30. Este libro, con explícita referencia a cuanto Aristóteles escribe sobre el nacimiento del Estado desde la familia, está presente en Menger (1883:175-177).
} 


\section{Escribe Menger:}

"Quien quiere llegar a una comprensión teórica de estos fenómenos humanos más complejos (...) debe extraer sus verdaderos elementos (...) e intentar indagar las leyes, según las cuales la economía social deriva de aquellos individuos (...). El objetivo más importante del camino teórico exacto es reconducir los hechos más complejos a los elementos más simples (...). Toda teoría, de cualquier signo, sea cual sea el grado de rigor del conocimiento al que quiere llegar, tiene el objetivo de hacernos conocer los fenómenos concretos del mundo real como manifestación de una cierta uniformidad en la sucesión de los fenómenos, lo que equivale a decir genéticamente (...). Este elemento genético es inseparable del concepto de ciencia teórica"4.

La atención debe centrarse en dos términos: el primero, en Aristóteles, .es "composición", que debe relacionarse con el método "compositivo" mengeriano; el segundo, en Menger, es "reconducir" (zurückführen), que no puede ser sustituido por "reducir" (lo que comportaría un procedimiento racionalista y no "genético"), o por "resolver". La importancia de tales términos es debida al hecho de que son

\footnotetext{
${ }^{4}$ Menger (1883: 70). He traducido "zurückführen" por reconducir y no por "resolver" o "reducir".

${ }^{5}$ En la traducción inglesa de Menger (1883: 93), zurückführen es traducido por to reduce (y no se trata de un ejemplo aislado; otro caso es la traducción de Wesen por nature). Desde esta traducción, en un ensayo que ha tenido una gran influencia, Watkins (1952: 29) escribe que para el individualismo metodológico el objetivo del científico social es continuar la investigación sobre los fenómenos sociales hasta que no sean reducidos a términos psicológicos. Esta definición puede ser considerada el acto de nacimiento de la interpretación "reduccionista" del individualismo metodológico. Esta será retomada por Nagel (1961: 541). La conclusión a la que llega este último es que "el individualismo metodológico se adhiere a una tesis fáctica (...) concerniente a la reducción de todos los enunciados acerca de los fenómenos sociales a una particular clase de enunciados ("psicológicos") sobre la conducta humana individual" (1961: 557). Watkins, en los ensayos citados, no alude a Menger, sino a Weber, Hayek y Popper, y cuando lo cite -en Watkins (1976), haciendo una revisión del individualismo metodológico como formulado en sus escritos de los años cincuenta- no se referirá al pasaje de Menger citado, sino a la página 152 de la traducción inglesa donde se habla de la moneda como "fenómeno social" (Watkins, 1976: 711). El hecho es que se trata de la traducción inglesa. Y la sucesiva observación, véase la página 716, según la cual la expresión individualismo metodológico "fue inventada por Ludwig von Mises". A su vez Nozick (1977:353) -en otro ensayo que ha tenido mucha influencia- escribe que "el individualismo metodológico asegura que todas las teorias de las ciencias sociales son reducibles a las teorias sobre la acción humana individual, más que a una serie de condiciones secundarias que especifican las condiciones en las que el individuo actúa", y en la respectiva nota (n. 2, pp. 385) añade que "un buen ejemplo standard de reduccionismo es el elaborado por Nagel". Se trata de un buen ejemplo de la confusión -o bien uno de los motivos de discusión-sobre el tema del individualismo metodológico. Naturalmente, en el capítulo del volumen de Nagel indicado por Nozick, no se cita a Menger y tampoco el párrafo dedicado al individualismo metodológico (véase pp. 550 y ss.). Sólo se citan a Mises y Hayek y, por lo que se refiere al individualismo metodológico, se plasma la citada definición de Watkins. Sobre la concepción del individualismo metodológico como "reduccionismo" en estos autores, véase Galeotti (1987: 216-
} 
indicadores del contexto filosófico aristotélico en el que se desarrollaba la reflexión mengeriana. Tales textos pueden ser valorados desde un análisis filosófico de la obra Untersuchungen; y ello induce a retener que los errores sobre el concepto de "método compositivo" derivan de una insensata comprensión de la concepción mengeriana del origen y de las leyes evolutivas de las instituciones sociales.

Que Menger tenga en la filosofía clásica su punto de referencia puede contemplarse en las páginas iniciales de la obra, donde se afronta el problema de la clasificación de las ciencias económicas. En todo su conjunto, Menger individúa tres grupos, elaborando de esta forma un modelo de distinción más próximo a la tradición aristotélica que a los modelos que le son contemporáneos: “(...) en primer lugar, las ciencias históricas (la historia y la estadística) de la economía, que deben indagar y representar la esencia y las relaciones de los fenómenos bajo el aspecto individual; en segundo lugar, la economía teórica, que debe indagar y representar la naturaleza y las relaciones de los fenómenos económicos (las leyes) bajo el aspecto general; en fin, en tercer lugar, las ciencias prácticas de la economía, que deben indagar y representar los criterios fundamentales del obrar económico, en relación cambio de las condiciones generales (la política económica y la ciencias de las finanzas)"'6 (Menger, 1883: 24-25).

Esta clasificación, confirmada en Untersuchungen (1883: 166-169), y en Die Irrthümer (Menger, 1884: 47-59), aparecerá, con alguna modificación, en Grundzüge einer Klassifikcation der Wirtschaftswissenschaften. Aquí Menger articula las ciencias en: 1 . Ciencias históricas de la economía: estadística económica e historia económica; 2. Morfología de los fenómenos económicos; 3. Teoría económica; 4. Economías prácticas o aplicadas (Menger, 1889: 199-200); e individúa el error de la Escuela histórica de los economistas alemanes en la deducción de normas del obrar práctico desde una equivocada concepción de la ciencia económica. Para obrar bien es necesario que las "ciencias económicas prácticas" encuentren su inspiración en una "teoría económica" no errónea. Por el contrario, si se fundamenta en una falsa concepción de la vida humana, toda política social estaría condenada al fracaso (Menger, 1884: 44-45; 1889: 208218) ya que produciría un número notable e incontrolable de consecuencias no deseadas que terminarían por hacer banales las premisas iniciales.

Tal clasificación de las ciencias es fundamental para comprender la teoría de las consecuencias no intencionales de las acciones humanas y de sus productos. Dicho en otros términos, la relación entre la acción humana, las ciencias prácticas y las leyes exactas. En efecto, las ciencias prácticas deben enseñar "los principios fundamentales según los cuales los fines económicos de las economías individuales

220), Rainone (1990: 169-225). Una interesante perspectiva respecto a la relación Menger-Hayek puede encontrarse en Antiseri (1987: 11-73).

${ }^{6}$ Sobre el tema de la clasificación de las ciencias véase también Menger (1889). 
(según las circunstancias) pueden realizarse de forma más completa" (Menger, 1883: 166-168); pero no pueden existir sin las ciencias teóricas; y, si se fundamentan en una errada configuración, dan lugar a políticas sociales destinadas al fracaso. Por tanto, se puede pensar en las consecuencias no deseadas como fruto del límite cognitivo de las ciencias prácticas - que no son "ciencias en el sentido estricto de la palabra", sino estudios "científicos" (Menger, 1889: 205)-: al producto de la ignorancia de las leyes exactas elaboradas a continuación de las acciones humanas.

Entre Aristóteles y Menger hay, sin embargo, una diferencia que no se debe olvidar. Si para Aristóteles la "naturaleza" constituye un orden conocer y que hay que secundar, para Menger, y para los sucesivos espontáneos de las Escuela Austriaca, la premisa del conocimiento del mundo viene dada por el hecho de que es un producto - aunque sea involuntario - de una actividad humana inspirada en la finalidad y en los conocimientos subjetivos y limitados. El mundo, en otras palabras, se configura como el resultado de la escasez de bienes y de conocimientos: de la lucha entre necesidades ilimitadas y recursos limitados. Hayek quiere superar la distinción entre artificial y natural en la clasificación y en el estudio de los fenómenos humanos, a favor de la definición fergusoniana como "el resultado de la acción humana, pero no del diseño humano" (Hayek, 1973-79: 20-21). Si tal es el objeto de las ciencias sociales teóricas, nos encontramos más allá de todo aristotelismo. En el caso de los Austriacos, se trata de descubrir como nunca desde acciones individuales inspiradas en fines subjetivos y en conocimientos limitados se produce un orden.

\section{La construcción metodológica de una ciencia de la acción social}

El mérito de Hayek es haber conjugado este método con la tradición individualista de la filosofía política anglosajona, y de haber, con el ensayo Individualism: True and False (Hayek, 1948: 1-32), enriquecido esta última con la teoría mengeriana del origen de las instituciones sociales, que considera más exhaustiva que la de Ferguson, Mandeville, Hume, Smith o Burke.

Por estos aspectos, que permiten una superación de la dicotomía natural-artificial en las ciencias sociales teóricas, y la asimilación del modelo evolucionista genético de Menger con el indeterminismo de la tradición inglesa -como se muestra en Law, Legislation and Liberty y en The Fatal Conceit - , Hayek puede considerarse como un continuador de la tradición mengeriana ${ }^{8}$. Pero, más allá

\footnotetext{
${ }^{7}$ Hayek (1973-79: 22-24); Hayek (1988: 143-147). Sobre tal asimilación véase Shearmur (1986: 210-224).

${ }^{8}$ Ahora bien, conviene tener presente que desde el punto de vista de la explicación genética de las instituciones, el ligamen con la concepción mengeriana sólo es parcial ya que, respecto a esta
} 
a mostrar que el colectivismo, la teoría de la planificación económica y, más en general, las formas de intervención estatal, forman parte de la tradición racionalconstructivista en cuyo ámbito encuentra ubicación el mismo contractualismo. Desde esta perspectiva, el fracaso del socialismo, del intervencionismo y de la planificación económica aparecen conectados al hecho de tener una falsa teoría de la naturaleza de la sociedad y de haber querido edificar sobre tal base tanto una filosofía política que trasladaba al totalitarismo (entendido como degeneración del orden político), como un sistema económico que no podía ser eficiente.

Obviamente, como sistema explicativo, el individualismo metodológico puede utilizarse para explicar entidades sociales de tipo colectivista. Sin embargo, no es sólo un sistema explicativo, también forma parte de una teoria relativa al nacimiento, al desarrollo y a la dinámica de las instituciones sociales. El mismo evolucionismo hayekiano no es sólo un sistema explicativo, sino una teoría sistemática de la política. El liberalismo espontáneo es al final demasiado abierto, y no puede ser interpretado como una forma de conservadurismo, ya que se perdería el significado del "cambio subjetivista" y de la preeminencia del consumidor sobre el productor.

La conceptualización hayekiana del método individualista y "compositivo" de las ciencias sociales tiene como punto de partida consideraciones gnoseológicas sobre la relación entre mente humana y "datos", para llegar a la conclusión de que en el ámbito de tales ciencias es necesario evitar "la acumulación de datos que derivan en entidades abstractas, y tomar sistemáticamente las muestras de las concepciones desde las que los hombres son inducidos a la acción" (Hayek, 1952: 40). En tal afirmación conviene señalar que el individualismo metodológico aparece conectado a la teoría gnoseológica hayekiana que desarrolla en Economics and Knowledge (1937), en The Facts if the Social Sciences (1942), en The Use of Knowledge in Society (1945), además de en Scientism and the Study of Society (1942-44) y especialmente en The Sensory Order (1952).

De ahí la necesidad de comprender los fenómenos más complejos del vivir social partiendo de las acciones individuales y de sus resultados: "en las ciencias sociales son los planteamientos de los singulares los que constituyen los elementos primarios, estos sirven para reconstruir, a través de combinaciones, los fenómenos complejos, es decir, para reproducir los resultados de las acciones individuales, que son menos notorios". Tal método, apelando explícitamente a Menger, es definido como "compositivo o sintético". Y Hayek concreta que "estos llamados conjuntos, estos grupos de elementos estructuralmente conectados, sólo aprendemos a aislarlos de la totalidad de los fenómenos observados como resultado de los esfuerzos sistemáticamente realizados para instituir conexiones estables entre elementos dotados de propiedades comunes, y a construirlos y reconstruirlos partiendo de las propiedades conocidas de los mismos elementos. Es importante observar que el objeto de nuestra explicación no son los diversos tipos de creencias y planteamientos individuales: éstos son simplemente los elementos a partir de los cuales reconstruimos las posibles estructuras relacionales inter-individuales". 
En consecuencia, para la ciencia social, las acciones humanas y los acontecimientos del mundo natural se configuran como datos que deben ser ordenados. $\mathrm{Su}$ objeto, por tanto, son las acciones que dan vida a resultados no previstos, y al nacimiento de regularidades que son espontáneas. De lo que se deriva que "si los fenómenos sociales no manifestasen otro orden que el atribuido por una intencionalidad consciente, no habría lugar para una ciencia teórica de la sociedad y todo se reduciría exclusivamente (...) a problemas psicológicos. Solamente en la medida en que emerge cierto tipo de orden como resultado de la acción de los singulares, pero sin ser conscientes de lo que persiguen, se plantea el problema de su explicación teórica" (Hayek, 1952: 43).

El análisis de estos pasos nos lleva a pensar que la composición de las acciones individuales en un orden más complejo no sigue tanto - como en Menger- un "principio" o una "ley" natural, sino una evolución indeterminada de la que, si no existe como fundamento de la acción más que un principio subjetivo, puede resultar muy difícil prever los resultados (Boniolo, 1990: 165-168). Hayek plantea la hipótesis de que los individuos escogen los fines y los medios según la configuración asumida por los fines en la mente de los mismos individuos y según la adecuación reconocida a los medios correspondientes, es decir, a partir de la distribución social del conocimiento y de la experiencia poseída por todo singular agente. De esta forma, el orden social se produciría espontáneamente como producto involuntario de la interacción de las acciones individuales vinculadas a la consecución de los fines subjetivos.

El olvidar todo esto representa para Hayek el gran error del colectivismo metodológico, que considera "que el conjunto de hechos no es otra cosa que teorías provisionales, modelos construidos por la mente ingenua para explicar las conexiones existentes entre algunos de los fenómenos singulares que observamos". Tales "conjuntos sociales", por otra parte, no son "unidades naturales", sino "complejos diversos de fenómenos singulares, entre sí totalmente diferentes, pero que nosotros consideramos ligados el uno al otro de forma similar; son selecciones de ciertos elementos dentro de un cuadro complejo, realizadas a partir de una teoría sobre su coherencia". Y es por ello que la mente humana, a partir de las leyes del pensamiento comunes a todos los seres humanos, selecciona desde un criterio de coherencia racional o lógica, los elementos que sirven para la imputación causal. Para Hayek no se trata por tanto de llegar a la esencia de los fenómenos, a las "leyes naturales exactas" de la sucesión de los fenómenos (como sostenía Menger). Tales "conjuntos" "existen sólo si, y en la medida en que, es exacta la teoría que hemos elaborado sobre la conexión de las partes implicadas y que sólo podemos explícitamente enunciar a partir de un modelo construido sobre la base de tales relaciones" (Hayek, 1952: 62-64).

En The Sensory Order se aclara esta perspectiva. En tal obra, Hayek no habla de individualismo metodológico, ni de metodología de las ciencias sociales teóricas, sino que desarrolla el modo en que la mente humana se forma un orden y el modo 
en que tal orden interactúa con el mundo fenoménico. El tema es complejo y no puede ser afrontado con profundidad en estas páginas (Cubeddu, 1994). Pero de la constatación de que las obras metodológicas y la obra de psicología teórica son contemporáneas - y que en esta última la atención se centra en el modo en que se forma un orden a través de una clasificación contemporáneamente, pero no de análoga importancia, genética y cultural de las experiencias sensoriales referentes al mundo fenoménico, y sobre su evolución- se sigue que la atención de Hayek, en aquellos años, se centra en la formación del orden. En The Sensory Order es posible encontrar el fundamento neurofisiológico de las obras metodológicas y políticas. Éstas, en otras palabras, no pueden ser vistas de forma separada, ya que el propio Hayek no las entendía separadamente, sino sistemáticamente conectadas. De tal obra, a decir verdad, todo sería de extremo interés para nuestros fines. Pero debemos limitarnos a la cuestión del individualismo metodológico. De una atenta lectura, y en concreto del párrafo en que Hayek niega la posibilidad de poder "llegar a una unificación completa de todas las ciencias en el sentido de poder describir en términos físicos todos los fenómenos de los que se ocupan las ciencias" del individualismo metodológico, o bien de aquella interpretación dominante a partir de los ensayos de John Watkins (1952: 29; 1957: 100), y que culmina en la conocida definición de Ernst Nagel (1961: 541-542).

Aparentemente, la forma en que Mises trata al individualismo metodológico no se distancia del planteamiento mengeriano "de reconducir los hechos más complejos a sus elementos más simples". Aunque para Mises "todas las acciones son realizadas por los individuos y para una colectividad social que no tiene existencia y realidad más allá de las acciones de los miembros individuales. La vida de una colectividad es vivida en las acciones de los individuos que constituyen el cuerpo. No existe colectividad social concebible que no funcione por las acciones de cualquier individuo. La realidad de un todo social consiste en las acciones de los individuos que la componen. De esta forma, el camino para llegar al conocimiento de los intereses colectivos pasa a través del análisis de las acciones de los individuos"'

\footnotetext{
"Véase Hayek (1952a: 88). Hayek aclara cómo el uso que hace del término "físico" se distingue del "lenguaje fisicalista" de Neurath y de Carnap.

${ }^{12}$ En Mises (1962: 41), aludiendo al individualismo metodológico, Mises escribe: "Un principio del análisis histórico de la acción humana consiste en afirmar que todas las acciones pueden reconducirse a los individuos y que ningún método científico puede determinar la formas en que ciertos acontecimientos externos, susceptibles de descripción a través de los métodos de las ciencias naturales, generan en la mente humana determinadas ideas, juicios de valor y deseos. En tal sentido, el sujeto, que puede dividirse en las partes que lo componen, constituye al mismo tiempo el punto de partida y el dato último de todos los esfuerzos encaminados al análisis de la acción humana".
} 
Sin embargo lo que cambia, y de forma significativa, es la premisa mediante la que podemos comprender la forma en que las acciones individuales tendentes a la consecución de fines subjetivos producen un orden social que, en buena medida, es producto involuntario de su obrar. En Mises, el equilibrio entre intereses individuales y naturalidad de los fenómenos y del obrar, que caracterizaba al pensamiento de Menger, desaparece para dar lugar a un marcado subjetivismo de la acción humana, subjetivismo que puede sintetizarse en las afirmaciones siguientes: "todos los valores humanos son ofertas a elegir" y "vivir es para el hombre el resultado de una elección, un juicio de valor" (Mises, 1949: 3-19). La premisa del conocimiento no es más que la naturalidad del obrar contemplada desde un fin, o bien la existencia y el descubrimiento de leyes universales a priori que regulan los resultados de la misma acción. A las leyes naturales exactas le sustituyen leyes a priori, consideraciones lógicas que tienen validez universal, mediante las que el individuo trata de dar un orden subjetivo al mundo.

Me parece inútil insistir en el hecho de que nos encontramos ante unas premisas filosóficas diferentes a las de Menger y Hayek, que, en adelante, no serán reclamados por Mises.

En los años cuarenta, también Popper elabora su propia concepción del individualismo metodológico, aludiendo explícitamente a Hayek y a Menger. En Intellectual Autobiography, Popper señala que su objetivo es "generalizar el método de la teoría económica (teoría de la utilidad marginal) para que pueda ser aplicada al resto de ciencias sociales teóricas" (Popper, 1976: 121). Y en The Poverty of Historicism, cuando aborda "la posibilidad de adoptar en las ciencias sociales el método que podemos llamar de construcción lógica o racional, o quizás el método del cero", escribe que "el método descrito parece coincidir en parte con el método que el profesor Hayek, siguiendo a Menger, llama compositivo" (Popper, 1944-45: 125-126). Como ejemplo de tal método, Popper trae de nuevo "el parangón entre comportamiento real (...) y comportamiento modelo que debemos esperarnos a partir de una pura lógica de la elección, como ha sido descrita por las ecuaciones de la economía". Aún más, cuando especifica que "ni el principio del individualismo metodológico, ni el del método cero para construir modelos racionales, (...) parecen comportar la adopción de un método psicológico (...), la psicología no puede ser considerada el fundamento de todas las ciencias, sino una ciencia social entre tantas" (Popper, 1944-45: 126), parece que Popper considera propia la teorización del rol de la psicología típica de la teoría del valor subjetivo.

Estos temas también pueden encontrarse en World 3, punto de llegada de la reflexión popperiana sobre la metodología de las ciencias humanas teóricas y sobre la naturaleza de sus objetos. Una reflexión que se había iniciado con el individualismo metodológico y con el zero method, y que, a través de la lógica de la situación, el análisis situacional y el principio de racionalidad, desemboca en el World 3 (Popper, 1976: 121). Este último tiene por objeto "sistemas teóricos", problemas, teorías científicas, instituciones sociales. Sus objetos "son nuestras 
R I S

REVISTA INTERNACIONAL DE SOCIOLOCIA

№ 34, Enero-Abril, 2003

MANUEL HERRERA CÓMEZ

construcciones, aunque no siempre sean el resultado de una producción proyectada por los singulares individuos" (Popper, 1977: 55). Pero su característica principal, más allá de la realidad, es la autonomía. En cuanto "producto no planificado de las acciones humanas", el World 3 trasciende a sus creadores (Popper, 1972: 215-216) para convertirse en el mundo de los "sistemas teóricos", de los "problemas", de las "situaciones problemáticas", de la discusión crítica y de su estado (Popper,1972: 151). A él, por tanto, nos referimos para comprender y explicar los fenómenos y los acontecimientos del mundo real a través de consideraciones lógicas que se ligan al análisis situacional. Es decir, a un "cierto tipo de explicación de cualquier acción humana que nos alude a la situación en que se encuentra el agente"; o bien, a "una reconstrucción idealizada de la situación problemática en la que el agente se ha encontrado", y que tiene el fin de hacer "comprensible la acción (o racionalmente comprensible): es decir adecuada a la situación tal y como él la veía. Este método de análisis situacional puede ser descrito como una aplicación del principio de racionalidad" (Popper, 1972: 235).

\section{LIBERALISMO Y COMUNITARISMO}

\section{La filosofía política de la Escuela Austriaca}

La exposición de la filosofia de las ciencias sociales del liberalismo austriaco puede ser útil para aclarar las diferencias entre ésta y otras corrientes de la filosofia política contemporánea. Para ilustrar la relación entre tal tipo de liberalismo y el comunitarismo nos podemos preguntar el porqué de la insistencia, por parte de los filósofos políticos contemporáneos que se declaran liberales, en la necesidad de un fundamento moral de las ciencias sociales y del mismo liberalismo. La respuesta a este interrogante puede ser útil para aclarar y comprender las relaciones entre las dos tradiciones de pensamiento.

Al abordar el individualismo metodológico hemós revelado las premisas gnoseológicas de una —quizás la más importante- de las corrientes liberales de la filosofia política contemporánea. Sin embargo, quien conozca, aunque sea superficialmente, las obras de los pensadores actuales que se llaman liberales (aparte de James Buchanan y Robert Nozick), no tardará en darse cuenta de que las referencias a la Escuela Austriaca son escasas y poco significativas. Obviamente, el discurso sobre la relevancia del liberalismo de la Escuela Austriaca en la filosofia política contemporánea no puede, en esta ocasión, ser afrontado en profundidad.

Los "austriacos", a diferencia de otros filósofos políticos contemporáneos (por ejemplo John Rawls), no se plantean el problema de una fundamentación moral del liberalismo, ni consideran que sea un elemento importante. $Y$ no es porque no consideren que la moral y la religión no sean importantes en el ámbito 
de una filosofia de las ciencias sociales y de la filosofia política - baste pensar en la importancia de esta cuestión en la última obra de Hayek, The Fatal Conceit (1988)-, sino porque consideran que una justificación ética de la política significa subordinar la política a la ética. En función de los límites del conocimiento humano, y del hecho de que tales límites actúan en cada una de las esferas en las que es posible investigarla, el intento de justificar éticamente la política se presenta problemático. La ética, dicho en otros términos, no puede ser el fundamento de un orden. Un orden es un orden: un fenómeno que puede ser investigado teóricamente y que puede ser valorado teórica y prácticamente, pero no se dice nada de que el juicio dependa de la bondad de sus premisas éticas. La intersección de las diferentes esferas, y de los efectos imprevistos que una esfera puede tener sobre las otras, pueden configurar un orden final que no tiene nada que ver con sus premisas iniciales.

En realidad, lo primordial para los "austriacos" es comprender cómo la intersección de las diferentes esferas de la acción humana contribuye a la formación de un orden. La propuesta política de este tipo de liberalismo consiste en una respuesta a los interrogantes clásicos de la filosofía política: cuál es el mejor régimen político, y si en él deben gobernar los hombres o las leyes. En otras palabras, estamos ante el problema del poder y de su relación con la filosofia, teniendo como punto de partida la convicción de que el poder y la investigación filosófica son conceptos opuestos y, en consecuencia, que el poder es un mal -aunque indispensable para preservar la existencia de una convivencia civil- que es necesario limitar, controlar eficazmente y, si es posible, reducir. Se ubica, por tanto, en la estela de la tradición clásica del liberalismo, aunque consciente de que las tradicionales premisas escogidas por el constitucionalismo liberal para controlar el poder han fracasado y es necesario inventar otras más eficaces. La inserción de este tipo de liberalismo en la más amplia tradición de la filosofia política que defiende la necesidad de un gobierno de la ley permite ver con mayor claridad lo que le distingue de otras filosofias políticas contemporáneas, también de aquellas que, llamándose liberales, atribuyen al Estado el objetivo de remover las causas materiales de la desigualdad social y de realizar de esta forma la igualdad y la libertad. Diversamente, los "austriacos" asientan la libertad en la diversidad entre los hombres.

El planteamiento conservador que se le atribuye, planteamiento que sería más correcto definir como anti-estatalista, consiste en valorar los cambios y las propuestas de cambio a la luz de los incrementos de poder estatal que, más o menos voluntariamente, pueden producir. No se trata por tanto de un planteamiento moral, sino de la constatación de la no reconciliación entre poder y filosofía y de la elección a favor de una libre investigación filosófica, desde la que se puede hacer emerger, a través de la libertad de discusión y crítica, la mejor solución para los problemas sentidos como comunes. Por otra parte, los límites de la conciencia humana no son sólo un atributo de súbditos y ciudadanos, también son los 
límites propios de todo ser humano: incluso de aquéllos que, con cualquier título, detentan el poder. En consecuencia, limitando los poderes de los gobernantes se puede limitar la posibilidad de que sus decisiones erróneas tengan consecuencias negativas en el conjunto de la sociedad. Desde otro punto de vista, también es posible considerar esta variedad del liberalismo como un intento para limitar los efectos negativos de las decisiones humanas a través de una rígida distinción entre una esfera privada que concierne a los fines, y una esfera pública que concierne a las reglas de conductas universalizables.

Si tenemos presente este tema, también podemos constatar fácilmente cuáles son las razones de las diferencias entre este tipo de liberalismo y otras filosofias políticas marcadas por una impronta liberal, democráticas, comunitarias, socialistas. Incluso se puede constatar que en el caso de los "austriacos" se nota la ausencia del proceso típicamente moderno, instaurado desde Maquiavelo, que ha hecho del Estado y de sus objetivos el principal sujeto de la política. Ahora bien, quizás - y limitada a la esfera de la reflexión filosófico-política-podemos afirmar que la época que ha privilegiado al Estado sobre la sociedad (entidad natural de la dinámica política), la época en que todos los modos han intentado "legitimar al bastardo", muestra todos sus límites, y que es necesario pensar en formas de convivencia política en cuyo ámbito el sujeto principal, y también el decisivo, no sea ya el Estado.

Las limitaciones y errores del conocimiento humano se convierten así en la premisa de una filosofía política entendida como investigación del mejor régimen político. Su fundamento es siempre la exigencia de evitar tanto los cambios bruscos que puedan derivar en la crisis del sistema de informaciones que permite la previsión de los resultados de las acciones individuales, como la creación de concentraciones de poder que puedan orientar tales intersecciones de informaciones hacia fines específicos o hacia la salvaguardia de intereses.

Dicho en otros términos, se trata de conservar la distinción entre fines y reglas, confirmando que la consecución de los primeros debe realizarse a través de los individuos, y que al Estado exclusivamente compete el mantenimiento - pero no la creación - de las reglas de comportamiento universalizables. Donde esta distinción no es válida, es decir, donde el Estado se ocupa de la consecución de los fines - quizás justificándolo con la necesidad de alcanzar objetivos muy justificables desde un punto de vista ético- y de la creación de reglas - la moderna actividad legislativa que hace depender el derecho de una decisión mayoritaria - nos encontramos, según esta tradición, fuera de la filosofia política liberal. En esto consiste la distinción entre liberalismo y democracia, que se configuran como dos respuestas diversas al problema de preferir un gobierno de las leyes o un gobierno de los hombres.

Si se contemplan las diversas tendencias de la filosofia política contemporánea desde esta perspectiva, la distinción, más allá de aquella apropiación de conceptos o expresiones con la que, muy a menudo, se produce una inútil e inmotivada 
confusión, aparecerá más clara. Desde el punto de vista teórico, entre estas dos alternativas no es posible la conciliación, tan sólo ocasionales intersecciones que, sin embargo, deben ser contempladas y valoradas en su carácter ocasional y casual. También en este caso es válida la afirmación tertium non datur. Y resulta incluso muy difícil de comprender por qué buscarlo.

También se tienen mayores indicaciones respecto al tema inicial del individualismo metodológico, que coincide con el objetivo principal de las ciencias sociales: diseñar las repercusiones no-intencionales de las acciones humanas sabiendo que toda acción que tenga lugar en régimen de escasez está sujeta al posible fracaso.

\section{El sistema político en acción: la relación medios-fines}

Llegados a este punto, sin embargo, no se puede ya eludir el problema consistềnte en indagar la condiciones de un orden. Para tratar este problema desde la perspectiva de la Escuela Austriaca sería oportuno decir algo sobre la relación entre la teoria de los valores subjetivos y las tesis expuestas por Hayek en The Sensory Order. Tal obra puede ser vista como una fundamentación neurofisiológica de la teoría de los valores subjetivos. En ella, Hayek sostiene que la valoración de los datos sensoriales por parte de la mente humana tiene lugar a través de un complejo sistema fisiológico que inserta el "dato" en un flujo de impulsos sensoriales que son clasificados y valorados por la mente humana a partir de una estructura genética (más o menos común a todo el género humano) y de la experiencia adquirida. Los "datos", en otras palabras, son insertados en clasificaciones pre-existentes y valorados, pero teniendo en cuenta que todo nuevo "dato" tiene la posibilidad de cambiar los esquemas clasificatorios precedentes.

Desde esta perspectiva, un orden político es análogo a un orden sensorial: consiste en un modelo para prever los resultados de las informaciones que van llegando a la mente. De igual forma que la mente humana clasifica las informaciones (datos sensoriales) para tener un marco - siempre provisional- de las informaciones útiles para actuar según los fines, y lo modifica cuando resulta que es ineficaz, un orden político es un sistema complejo que trata de calcular los resultados que las singulares acciones individuales puedan tener en la consecución de los fines, $y$ tiende a descartar los comportamientos que impiden alcanzar tales fines, o bien los reformula.

La ciencia económica es solamente aquella rama de la filosofia de las ciencias sociales que, gracias a la teoría de la utilidad marginal y a la consiguiente teoría de los valores subjetivos, se da cuenta de las modalidades del proceso a través del cual se produce un orden. Un orden puede ser tanto un proceso de mercado, como un más complejo sistema social, en el que entran en contacto las diversas esferas de la acción humana: económica, ética, política, jurídica, lingüística, etc. Por tanto, los exponentes de la Escuela Austriaca se dan cuenta de un proceso 
RIS

REVISTA INTERNACIONAL DE SOCIOLOCIA

$N^{0} 34$, Enero-Abril, 2003

MANUEL HERRERA CÓMEZ

que tiene lugar en cualquier campo de la actividad humana. Su insistencia en la importancia de una teoría de la acción humana no errónea está conectada a la conciencia del hecho de que, por el principio de las consecuencias involuntarias, toda esfera puede tener una función determinante para la creación de un orden, y que el resultado final de un proceso social es en buena medida imprevisible debido a la limitación y debilidad del conocimiento humano y de sus instrumentos clasificatorios.

En el resto de las filosofias de las ciencias sociales, que sin embargo se proclaman liberales, todo esto no se afronta con la misma solidez teórica.

Desplazando el discurso hacia las teorías comunitarias, se puede observar que mientras los planteamientos de los "austriacos" se mueven desde la convicción de que todo deseo subjetivo no puede determinar el resultado final del proceso de orden, ni tiene el derecho de hacerlo, el discurso de los comunitaristas tiene el siguiente punto de partida: independientemente del resultado final, un estatus subjetivo debe mantenerse como tal y conservarse también en el resultado final del proceso de la catarsis. A los estatus subjetivos, en otras palabras, se les atribuye el privilegio de conservación independientemente de la funcionalidad del orden que se deriva. Obviamente, es necesario tener presente que un orden que deriva de status iniciales no modificables en el ámbito de un proceso de integración y de modificación de esferas y de comportamientos será un orden complejo, frágil y vinculado al mantenimiento de las condiciones iniciales. Al final, esto producirá un notable incremento de las controversias, o el dominio de un status, o de un grupo de status sobre otros, exclusivamente a partir de relaciones de fuerza o de poder.

En definitiva, se tendrá una situación en la que los individuos no tendrán valores y derechos en cuanto individuos, sino en cuanto pertenecientes a un status, y en el que el valor de los status estará en conexión con su relación con los status mayoritarios; con aquellos que, conquistadas las posiciones de poder, también tendrán la facultad de establecer que status subjetivos son compatibles con el resultado final del proceso social. El concepto liberal de rule of law es sustituido, en esta perspectiva, por un sistema jurídico basado en la pertenencia a un status.

Sin embargo, existe una complicación posterior debida a que una pluralidad de estatus no podrá ser separada de la existencia de un sistema de producción y de distribución de los recursos. Pero si éste es dominado por un grupo de intereses ligado a determinados estatus, la distribución de los recursos no podrá prescindir de sus intereses. En consecuencia, los estatus que no deberían converger o que fuesen conflictivos con los intereses de los status dominantes recibirán menos de cuanto subjetivamente esperan. El resultado será un incremento de la conflictividad debido a que el resultado final del proceso de distribución no estará ligado al agrado que los bienes producidos recibirán por los consumidores, sino a motivaciones políticas, étnicas o éticas atribuidas al proceso de distribución. El tema inicial de la salvaguarda de la diversidad se transformará en un intento de 
reconducir el proceso de integración social hacia la consecución de determinados fines o la salvaguarda de determinados intereses. Todo ello podrá ser aceptable desde un punto de vista étnico o ético, pero también podrá producir una conflictividad económica, social y política, y seguramente absorberá la condición jurídica fundamental de la igualdad.

Mientras en la tradición del rule of law la igualdad entre los individuos está garantizada por el imprevisible resultado del proceso, a través de las reglas de conducta vinculantes para todos, las cuales no imponen fines sino comportamientos para conseguir los fines subjetivos; en la tradición comunitaria la igualdad sólo será un dato de partida, pero no todos tendrán las mismas garantías respecto al resultado final porque no todos tendrán iguales posibilidades de influir en el resultado, en cuanto que faltará el instrumento neutro del mercado como sistema de transmisión de informaciones que cada uno utilizará como quiera. Si se tięnde hacia un resultado final del proceso, quien lo dirija tendrá indudablemente mayores oportunidades que quienes no lo hagan. En esto se puede medir la ventaja del sistema de rule of law, que tiende a evitar que una posición de momentánea ventaja se transforme en un poder de dirección hacia la consecución de fines o hacia la conservación de privilegios ligados a un status.

Desde este punto de vista también se puede medir la importancia atribuida por los "austriacos" al mercado, entendido como sistema de transmisión de informaciones neutral respecto a los fines, es decir, éticamente neutral. Concebir el mercado como un sistema para realizar fines comporta, en definitiva, si se lo valora por los resultados, numerosos inconvenientes. Quien decida los fines, en otras palabras, también tendrá la posibilidad de dirigir el proceso según los propios intereses, pero, teniendo un conocimiento limitado, no se ha dicho que el resultado final del proceso vaya a ser el esperado, ni, tampoco, el justo.

En definitiva, para comprender la filosofía política de la Escuela Austríaca, su concepción del liberalismo, y también su crítica al concepto de justicia social, es necesario tomar las muestras desde una interpretación de su filosofia de las ciencias sociales como extensión de la teoría de los valores subjetivos al resto de los fenómenos sociales.

Simplificando, podemos resumir tal teoría afirmando que en la vida social, en general, como en la vida económica, no existen valores objetivos, sino sólo valores subjetivos que individuos dotados de un conocimiento limitado atribuyen a bienes o informaciones en un régimen de escasez, incluso subjetivo. En consecuencia, como no existe un bien común, no existe un concepto de justicia, y mucho menos un concepto de justicia social, que pueda emerger desde una orientación para las acciones individuales y para la vida política en general.

El mercado, o bien el proceso de la catarsis, no es el mejor sistema para una equitativa distribución de los recursos, pero, por lo que se conoce, es el sistema más eficiente en la transmisión de informaciones. La catarsis no tiene necesidad de ninguna justificación moral. De tales informaciones los individuos que actúan 
RIS

en condiciones de libertad pueden hacer el uso que quieran. Sin embargo, los usos fraudulentos son penalizados por el sistema de la catarsis; en el sentido que tienen como consecuencia no deseada la expulsión de quien los activa desde el sistema de los intercambios recíprocos en condiciones de escasez. La catarsis es un sistema de intercambio de expectativas subjetivas que está detrás de la condición del recíproco respeto de cuanto se ha pactado. En un sistema complejo esto plantea el problema de las reglas universales de conducta que prescriben comportamientos para la consecución de fines individuales o colectivos. Estas reglas, en palabras de Oakeshott, constituyen el bien común de una asociación civil.

La ética, desde esta perspectiva, se configura como una esfera de la acción humana. Pero no es la esfera a la que hay que subordinar el resto, ni la esfera desde la que es posible valorar las otras esferas. La ética es una esfera de reflexión teórica como las otras, y sus enunciados, desde el punto de vista social y pôlítico, deben ser valorados exclusivamente desde el punto de vista de las consecuencias no intencionales que derivan de la aplicación práctica de sus enunciados. Por tanto, ningún primado de la ética. Ciertas elecciones éticas pueden tener consecuencias letales para una sociedad. Por otra parte, una sociedad ética no es una sociedad libre.

En consecuencia, el liberalismo austriaco no pretende ser un intento de encontrar una justificación ética a la sociedad liberal. Esto lo diferencia tanto de las tradicionales justificaciones de la sociedad liberal planteadas por los pensadores liberales del pasado, como de los más recientes intentos del liberalismo, por ejemplo los de Rawls, Nozick, Raz, Gauthier y otros.

Norman P. Barry, en On Classical Liberalism and Libertarianism, afirma que la cuestión de la ética de la libertad y de la justificación de los principios fundamentales de liberalismo es el problema más debatido en el ámbito de la tradición liberal y de la tradición libertaria. Sin embargo, como se ha dicho, Hayek no se plantea el problema de una fundamentación ética del liberalismo. Para él la libertad es un dato de hecho que deriva de la natural diversidad de los hombres. Algo que debe ser conservado para mantener tal diversidad, y por ello es necesario caminar hacia la búsqueda de un orden político mejor. De lo que se sigue que toda acción tendrá consecuencias de carácter positivo y de carácter negativo que podrán ser percibidas diversamente por los sujetos sobre los que tiene efectos. Pero la ética, con todo esto, no entra para nada.

El principal temor de Hayek es que el intento de perseguir o de realizar la justicia social pueda resolverse con un incremento del poder del gobierno o con tener este último como consecuencia no intencional. Por otra parte, no teniendo ningún fundamento gnoseológico en el sentido anteriormente expuesto, la teoría de la justicia social no estará en situación de alcanzar sus propósitos, pero se resolverá en una dinámica democrática, en un nuevo estado de guerra civil entre grupos sociales organizados que reclamarán el derecho de protección jurídica y política del propio estatus, lo que equivale a decir en una guerra entre grupos que tratarán de asegurarse posiciones privilegiadas en la distribución de los recursos. 
La escasez de los recursos determinará un estado de conflicto cuanto más escasos sean los mismos recursos; y cuanto más ineficiente sea el sistema económico, los conflictos serán más violentos.

Tal juicio se fundamenta en la constatación de que los teóricos de la justicia social no han elaborado una teoría económica que sirva de apoyo, de alguna forma, a su teoría política o moral. La parte económica de la teoría de la justicia social no es otra cosa que, en opinión de Hayek, una reformulación no original de las teorías económicas socialistas, intervencionistas, o un desarrollo de ciertos axiomas de la economía del bienestar. El hecho de que se apoye en una teoría económica errónea o inconsistente comporta, según Hayek, que la teoría de la justicia social no podrá conseguir sus fines, sino que se resolverá en un estado de perenne conflicto. Toda distribución igualitaria de recursos (económicos, servicios, información, cultura, etc.) tendrá resultados que podrán ser valorados diversamente por los sujetos interesados. En consecuencia, serán necesarias posteriores y constantes redistribuciones; lo que podrá realizarse sólo mediante un poder político progresivamente más fuerte y extenso. Esta situación también plantea el problema de cuánto las buenas intenciones morales están privadas de consecuencias indeseadas. Pero también es el resultado del intento de guiar una sociedad hacia la consecución de finalidades éticas que no pueden ser justificadas desde un conocimiento limitado.

Para concluir, no es como consecuencia de los escritos en los que Hayek ha considerado la polémica entre individualismo y comunitarismo, ni las recientes tendencias de la filosofia política y su insistencia en el tema de reconocimiento social, jurídico y político de las "diversidades"; pero, si que me he arriesgado a avanzar una conjetura: pienso que él habría tenido dificultades para ubicarse en una de las formaciones contrapuestas, ya que habría rechazado ambas. Sin embargo, imagino que habría interpretado la discusión como uno de los deletéreos resultados de aquella batalla que ha tenido como objeto la apropiación temática y los conceptos clásicos de la filosofia de las ciencias sociales y, como fin, dar las mismas interpretaciones que nada tienen que ver con su significado tradicional. $\mathrm{La}$ actual insistencia en el tema del "reconocimiento de las diversidades", en esta perspectiva, también se podría interpretar como el eclipse de la tradición del rule of law y, como fruto, la proposición, perseguida por la tradición de la justicia social, de considerar a los individuos no en cuanto sujetos de un ius cosmopoliticum, sino en cuanto pertenecientes a un estatus, en otras palabras, del intento de hacer derivar los derechos individuales y sociales desde un estatus y no del común sometimiento al rule of law; o bien de la creencia de que todo estatus, o toda expectativa subjetiva tiene derecho a una representación jurídica y política ${ }^{13}$.

\footnotetext{
${ }^{13}$ Personalmente creo que la tradición liberal tiene poco que ver con la tradición del classical liberalism, así como que el rule of law es la esencia de tres liberalismos, y comparto gran parte de las críticas que Gray (1993) dirige al fundamentalist liberalism.
} 


\section{RIS}

La respuesta al problema de si las soluciones avanzadas por las recientes tendencias de la filosofía política y moral pueden ayudar a la solución de los problemas económicos, políticos y sociales requiere tiempo. Sin embargo, recordando el famoso dicho que afirma "que de buenas intenciones está lleno el camino del infierno", en la actualidad sólo se puede llevar a cabo una reedición generalizada de las luchas que Hobbes había tratado de conjurar, y la aventura, para restablecer cualquier apariencia de orden, de un nuevo y más terrible Leviatán. Los menos pesimistas, sin embargo, podrán consolarse pensando en una novela de Luigi Pirandello: La patente.

\section{REFERENCIAS BIBLIOGRÁFICAS}

ALTER, M. (1990), Carl Menger and the Origins of Austrian Economics, San Francisco-Oxford, Westview Press.

ANTISERI, D. (1987), "Individualismo metodologico e autonomia della sociologia", en D. Antiseri, L. Infantino y G. Boniolo, Autonomia e medodo del giudizio sociológico, Roma, Borla.

BONIOLO, G. (1987), "Non prevedibilità e modelli sociologici desunti dalle scienze formali", en D. Antiseri, L. Infantino, G. Boniolo, Autonomia e metodo del giudizio sociológico, Roma, Armando.

CUBEDDU, R. (1986), "Dal metodo compositivo all'individualismo metodologico", Quaderni di Storia dell'Economia Politica, nº. 4, pp. 23-45.

(1993), The Philosophy of the Austrian School, Nueva York, Routledge.

(1994), "From the Sensory Order to the Social Order", Philosophy Politics Economics, PPE - Lectures, 10, Department of Economics, Universidad de Viena.

GALEOTTI, A. E. (1987), "Teoria politica e modi di spiegazione. Il caso dell'individualismo metodologico", en Veca, S. (comp.), Filosofia politica e societá. Utilitarismo e teoria della giustizia, Nápoles, Bibliopolis.

GORDON, S. (1991), The History and Philosophy of Social Science, Londres-Nueva York, Routledge.

GRAY, J. (1993), "What is dead and what is living in liberalism?", Post-Liberalism. Studies in Political Thought, Londres-Nueva York, Routledge.

MENGER, C. (1871), Grundsätze der Volkswirthschaftslehre, Viena, W. Braumüller. [Principios de economia politica, Madrid, Unión Editorial, 1997].

(1883), Untersuchungen über die Methode der Sozialwissenschaften und der politischen Oekonomie insbesondere, Leipzig, Dunker \& Humblot.

(1884), Die Irrthümer des Historismus in der deutschen Nationoalökonomie, o en Gesammelte Werke, J. C. B. Mohr, Tubinga, 1970, Bd. III. 
(1889), "Grundzüge einer Klassikikation der Wirschaftswissenschaften", Jahrbüchern für Nationalökonomie und Statistik, XIX, o en Gesammelte Werke , J. C. B. Morh, Tubinga, 1970, Bd. III.

(1923), Grundsätze der Volkswirtschaftslehre (Hrsg. K. Menger), Holder-Pichler-Tempsky Ag.

MISES, L. VON (1949), Human Action, New Haven, Yale University Press. [La acción humana, Madrid, Unión Editorial, 1995].

(1962), The Ultimate Foundation of Economic Science, Kansas City, Van Nostrand.

NAGEL, E. (1961), The Structure of Science, Nueva York, Harcourt, Brace \& World, Inc. [La estructura de la ciencia, Barcelona, Paidós, 1991].

NOZICK, R. (1974), Anarchy State and Utopia, Nueva York, Basic Books. [Anarquia, Estado y Utopia, México, FCE].

(1977), "On Austrian Methodology", Syntese, n 36, pp. 2.

POPPER, K. R. (1944-45), The Poverty of Historicism, Londres, Routledge. [La miseria del historicismo, Madrid, Alianza Editorial, 1999].

(1959), The Logic of scientific Discovery, Basic Books, Nueva York. [La lógica de la investigación científica, Madrid, Tecnos, 1985].

(1972), Objective Knowledge. An Evolutionary Approach, Oxford, Clarendon Press. [Conocimiento objetivo, Madrid, Técnos, 2001].

(1976), Unended Quest. An Intellectual Autobiography, Glasgow. [Búsqueda sin término, Madrid, Alianza Editorial, 2002].

(1977), The Self and Its Brain, Berlín-Heidelberg-Londres, Springer.

RAINONE, A. (1990), Filosofia analitica e scienze storico-sociali, Pisa, ETS.

SCHUMPETER, J. A. (1908), Das Wesen und der Hauptinhalt der theoritischen Nationalökonomie, Leipzig, Dunker\& Humblot.

SHEARMUR, J. (1986), "The Austrian Connection: Hayek's Liberalism and the Thought of Carl Menger", en Grassl, W. y Smith, B. (eds.), Austrian Economics: Historical and Philosophical Background, Londres, Croom Helm.

VON HAYEK, F. A. (1937), “Economics and Knowledge”, en Hayek (1948a).

(1942), “The Facts of the Social Sciences", en Hayek (1948a).

(1942-44), "Scientism and the Study of Society", Economica, N. S. IX.

(1945), "The Use of Knowledge in Society", en Hayek (1948a). 
(1948), "Individualism: True and False", en Hayek (1948a).

(1948a), Individualism and Economic Order, Londres, Routledge.

(1952), The Counter-Revolution of Science. Studies of the Abuse of Reason, Freee Press, Glencoe (III).

(1952a), The Sensory Order, Londres, Routledge.

(1967), "Kinds of Rationalism", en Hayek (1967a).

(1967a), Studies in Philosophy, Politics and Economics, Londres, Routledge.

(1973-79), Law, Legislation and Liberty, 3 vols, Londres, Routledge. [Derecho, legislación y libertad, Madrid, Unión Editorial, 1999].

(1978), "The Errors of Constructivism" y "Liberalism", en Hayek (1978a).

(1978a), New Studies in Philosophy, Politics, Economics and the History of Ideas, Londres, Routledge.

(1988), The Fatal Conceit. The Errors of Socialism, Londres, Routledge. [La fatal arrogancia: los errores del socialismo, Madrid, Unión Editorial, 1990]

WATKINS, W.N. (1952), "Ideal Types and Historical Explanation", British Journal for the Philosophy of Science, III.

(1957), "Historical Explanation in the Social Sciences", British Journal for the Philosophy of Science, VIII.

(1976), "The Human Condition: Two Criticism of Hobbes", en Cohen, R.S. y Feyerabend, P.K. (eds.), Essays in Memory of Imre Lakatos, Dordrecht, Riedel. 